\begin{tabular}{|c|c|c|}
\hline Beitr. Ent. & Keltern & ISSN 0005-805X \\
\hline $\mathbf{5 6}(2006) 2$ & S. $442-450$ & 15.12 .2006 \\
\hline
\end{tabular}

\title{
Erkenntnisse zum Lebenszyklus von Byctiscus betulae (LINNÉ, 1758) aus den Weinbauanlagen von Odobeşti, Kreis Vrancea / Rumänien
}

\section{(Coleoptera: Curculionoidea, Rhynchitidae)}

Mit 3 Figuren und 6 Tabellen

\section{Iuliana Antonie, Irina Teodorescu und Letiția Oprean}

\begin{abstract}
Summary
This study was conducted in Odobeşti (Vrancea) Vineyard Region. In this vine-growing ecosystem a mass outbreak of Byctiscus betulae L. which lasted for 4 years (2000-2003) was studied at regular intervals of 3-4 weeks.

Oviposition was investigated on 12 varieties of vine, by counting the leaf-rolls of all stages and the number of adults on six stems of each variety of vine. Total material comprised 728 leaf-rolls and 452 adult specimens. Research on the egg and larval stages was undertaken in 2000. From 766 leaf-rolls were extracted 534 healthy eggs; 392 parasite eggs; 179 neonate larvae; $1274 \mathrm{~L}_{1}$ larvae; $898 \mathrm{~L}_{2}$ larvae and $6 \mathrm{~L}_{3}$ larvae. The low percentage of $\mathrm{L}_{3}$ larvae led us to believe that the development of the last larval instar takes place in the ground. Experimental evidence for this was later obtained.

In 2000-2001 we made 65 searches in the soil between previously sampled stems of vines of the Aligote variety on Şarba Hill. No pupae of B. betulae could be found. In 2002 pupae could only be obtained in the laboratory. The first adult was reared under humidity controlled conditions a week after the first pupa was formed. The pupa is stenohygrous, formed only at approximately $50-55 \%$ relative humidity.
\end{abstract}

\section{Zusammenfassung}

Die Untersuchungen wurden im Weinbaugebiet (Vrancea) durchgeführt. Während vierer Jahre (2000-2003) wurde das vom Rebenstecher (Byctiscus betulae) stark belastete Gebiet in jeweils 3 bis 4 Wochen Abstand aufgesucht.

Der Befall wurde an 12 Weinsorten geprüft, wobei jeweils an 6 Stellen die Blattrollen und die Anzahl der Adulti gezählt wurden. Insgesamt wurden 728 Blattrollen und 452 Adulti ermittelt (am 05.05.2000). Zur Untersuchung der Ei- und Larvenstadien wurden im Jahre 2000 auf dem Odobeşti-Weingut, Şarba-Berg, 766 Blattrollen gesammelt, aus denen 534 normale Eier, 392 parasitierte Eier, 179 neonate Larven, 1274 $\mathrm{L}_{1}$-Larven, $898 \mathrm{~L}_{2}$-Larven und $6 \mathrm{~L}_{3}$-Larven gezogen wurden. Der reduzierte Anteil an $\mathrm{L}_{3}$-Larven lässt vermuten (bzw. bestätigt), dass die Entwicklung des letzten Larvenstadiums von Byctiscus betulae im Boden stattfindet.

In den Jahren 2000 und 2001 wurden 65 Bodenproben zwischen den Teststellen der Weinsorte Aligote am Şarba-Berg (Odobeşti, Vrancea) geprüft, aber keine Puppenhüllen von Byctiscus betulae gefunden. Solche wurden 2002 erst im Labor gefunden. Im Experiment wurde der erste Adultus im Feuchtegradienten eine Woche nach der ersten Puppe ermittelt. Die Puppe ist offenbar stenohygrisch, denn wir fanden sie ausschließlich bei ca. 50-55\% relativer Feuchte. 


\section{Einführung}

Besonders aus Weinbaugebieten sind die sogenannten Rebenstecher oder Rebstichler (Byctiscus betulae L.) bekannt, die zu den Curculionoidea gehören, einer Überfamilie, die mehr als 50000 Arten umfasst. Es waren hauptsächlich drei Faktoren, die uns bestimmt hatten, diese Art zu untersuchen:

- das Fehlen eingehender Studien zur Biologie und Ökologie dieser Art in der rumänischen Fachliteratur;

- Besonderheiten der Ethologie dieser Art im Vergleich zu anderen Coleopteren;

- der ernst zu nehmende ökonomische Faktor, denn dieser Schädling richtet in den Weinbaugebieten beträchtlichen Schaden an.

Die erste Erwähnung dieser Art in Rumänien geht auf E. A. BIELz (1851) zurück, der die ersten Coleopteren-Kataloge für Transsilvanien / Siebenbürgen herausgebracht hat. In ihnen werden 16 Arten von Rhynchitiden angeführt, darunter auch Byctiscus betulae. Der Autor war damals bestrebt, möglichst viele Arten zu identifizieren und anzuführen, so dass er den Fundorten wenig Aufmerksamkeit schenkte und diese deshalb nicht registriert hat.

Im Jahr 1869 veröffentlichte C. Fuss das "Verzeichnis der Käfer Siebenbürgens nebst Angabe ihrer Fundorte” im 3. Heft des 1. Bandes der Zeitschrift „Archiv des Vereins für Siebenbürgische Landeskunde”, herausgegeben in Kronstadt (Braşov). Auch hier wird unter den 21 Arten von Rhynchitiden Byctiscus betulae angeführt.

Die größte faunistische Liste der Rhynchitidae und Attelabidae, nämlich 25 Arten im gleichen Areal, wird 1912 von K. PETRI veröffentlicht; er bringt die vorausgehenden Klassifizierungen von BIELz, Fuss sowie anderer Autoren auf den neuesten Stand der Wissenschaft.

\section{Material und Methode}

Die vorliegende Untersuchung wurde im Weingut Odobeşti im Landkreis Vrancea durchgeführt. Die Beobachtungen dauerten über 4 Jahre an (2000-2003); sie wurden in regelmäßigen Abständen von 3-4 Wochen durchgeführt.

Als Sammelmethoden im Freiland kamen zum Einsatz:

- Einsammeln von Byctiscus betulae von Hand;

- Sammeln und Pressen von befallenem Blattwerk, von Trieben und Knospen, die angefressen oder eingerollt waren;

- ein Fangnetz, bestehend aus einem Metallrahmen von $30 \mathrm{~cm}$ Durchmesser mit wenigstens $60 \mathrm{~cm}$ langem Seidensieb (Müllergaze) sowie Teleskopgriff von $80 \mathrm{~cm}$ Länge. Die in dieses Netz gefallenen Insekten werden in einem mit Äther oder Chloroform gefüllten Glasgefäß getötet.

- Sondierung im Erdreich im nach Larven und Puppen. - Diese Untersuchungen auf der Fläche eines Quadrats von $25 \mathrm{~cm}$ Seitenlänge gehen $30 \mathrm{~cm}$ tief. Die Funde werden in 70 \%igem Alkohol konserviert. Die Sondierungen werden in der Diagonale des Untersuchungsgebiets im Abstand von $10 \mathrm{~m}$ durchgeführt.

- Die Winter-Falle. - Ein Kasten von 50 x $50 \mathrm{~cm}$ wird mit der Öffnung auf die Erde gestellt, eine Schicht vermodertes Blattwerk untergeschoben und vom Herbstende 
bis zum 15. März bzw. 1. April liegen gelassen. Danach wird es mitsamt der anhaftenden feinen Erdschicht herausgehoben und im Labor untersucht.

- „Betten für das Vorpuppenstadium”. - Eine $20 \mathrm{~cm}$ tiefe Erdgrube wird mit Leinwand oder Plasikfolie ausgelegt. Darauf werden Blattrollen gebracht, die Larven der Stadien $\mathrm{L}_{2}$ und $\mathrm{L}_{3}$ enthalten. Die Grube wird mit Erde und vermodertem Blattwerk ausgefüllt.

Im Labor wurde eine eigene Methode angewendet.

- Einrichtung eines Feuchtigkeits-Gradienten für das Puppenstadium von Byctiscus betulae. - Ein Plastik-Gefäß von 100 x $80 \mathrm{~cm}$ Grundfläche wird mit einer $8 \mathrm{~cm}$ dicken Erdschicht belegt und in einer Neigung von 20-30 aufgestellt. Vom oberen Ende ausgehend, wird die Erde befeuchtet, worauf sich die Feuchtigkeit etwa wie folgt verteilt: an der tiefen Stelle werden 100\% gemessen, in der Mitte 50-55\%, im oberen Teil 2-3\%. Das biologische Material, meist gleichzeitig junge Larven $\left(\mathrm{L}_{1}, \mathrm{~L}_{2}\right)$ und von erwachsenen Tieren geformte Blattrollen. Die Larven verlassen die Blattrollen und siedeln sich nach Vorliebe an. Mit Hilfe dieser Methode ist es gelungen, neben Puppen in Erdzellen gleichzeitig die letzte Larvenstufe sowie Imagines zu „züchten“. Die Temperatur im Labor betrug $18-23{ }^{\circ} \mathrm{C}$.

\section{Ergebnisse und Diskussion}

Der Ort der Eiablage von Byctiscus betulae wurde an 12 Rebensorten untersucht (am 05.05.2000). Dabei wurden die Blattrollen sowie die Imagines bei jeweils 6 Rebstöcken gezählt, was 728 Blattrollen und 452 adulte Exemplare ergab. Die folgende Übersicht spiegelt das Ergebnis (Tabelle 1).

Tab. 1: Das prozentuale Vorkommen von Byctiscus betulae bei verschiedenen Rebsorten (am 05.05.2000).

\begin{tabular}{|c|c|c|c|c|c|}
\hline & Rebsorte & $\begin{array}{l}\text { Anzahl Blattrollen } \\
\text { an } 6 \text { Weinstöcken }\end{array}$ & $\%$ & Zahl der Imagines & $\%$ \\
\hline 1 & Plăvaie & 184 & 25,24 & 111 & 24,56 \\
\hline 2 & Milcov & 102 & 14,01 & 83 & 18,37 \\
\hline 3 & Şarba & 98 & 13,46 & 66 & 14,60 \\
\hline 4 & Merlot roşu & 72 & 9,89 & 49 & 10,84 \\
\hline 5 & Aligoté & 54 & 7,42 & 45 & 9,96 \\
\hline 6 & Fetească Regală & 52 & 7,14 & 25 & 5,53 \\
\hline 7 & Băbească Neagră & 50 & 6,87 & 23 & 5,08 \\
\hline 8 & Codană & 46 & 6,32 & 19 & 4,20 \\
\hline 9 & Fetească Neagră & 28 & 3,85 & 16 & 3,54 \\
\hline 10 & Chasselas & 22 & 3,02 & 10 & 2,21 \\
\hline 11 & Pinot Noire & 20 & 2,75 & 5 & 1,11 \\
\hline \multirow[t]{2}{*}{12} & Risling Italian & 0 & 0 & 0 & 0 \\
\hline & Total & 728 & $100 \%$ & 452 & $100 \%$ \\
\hline
\end{tabular}


Die Übersicht zeigt bei der Sorte Plăvaie die höchste Blattrollen-Bildung und den höchsten Byctiscus-Befall, gefolgt von Milcov, während die drei anderen Rebsorten im Schädlingsbefall einander gleichen. Nur geringen Befall zeigen Chasselas und Pinot Noire, während der Italienische Riesling sich als schädlingsfrei darstellt.

Unseren Untersuchungen zufolge ist die chemische Zusammensetzung der Rebenblätter kein bestimmender Faktor für den Byctiscus-Befall. Dagegen kommt der Blattform für die Eiablage eine besondere Bedeutung zu: der Käfer bevorzugt deutlich ganzrandige Blätter ohne Vertiefungen, Ausbuchtungen, ohne Rillen und Flaum. Je weniger das Blatt im diesem Sinn gegliedert ist, desto leichter lassen sich damit die besagten Blattrollen drehen.

Außer den gesammelten 6341 Blattrollen von Weinstöcken wurden weitere Brutstätten von Byctiscus wie folgt ermittelt: 282 von Linden, 7 von Birnbäumen sowie 13 von Pappeln (in den Jahren 2000-2002).

Die Tabelle 2 verauschaulicht die relative Häufigkeit des im Jahr 2000 (05.05. und 25.05.2000) gesammelten Gesamtmaterials vom Şarba-Berg:

- Die Zahl der untersuchten Blattrollen betrug im Jahr 2000766 Stück;

- die Blattrollen waren aus 1-5 Blättern zusammengerollt;

- die normale Eiablage betrug 16,26 \%; parasitierte Eier 11,94 \%; frischgeschlüpfte Larven 5,45 \%; Larven $\mathrm{L}_{1} 38,83 \% ; \mathrm{L}_{2} 27,35 \% ; \mathrm{L}_{3} 0,18 \%$. Das geringe Vorkommen der Larve $\mathrm{L}_{3}$ bestärkt uns in der Annahme, dass die Entwicklung des letzten Larvenstadiums von Byctiscus betulae in der Erde erfolgt.

In geringer Entferung des von Byctiscus betulae befallenen Weinguts Odobeşti befinden sich Linden mit starkem Schädlingsvorkommen; am 05.05.2000 wurden dort 44 Blattrollen eingesammelt, deren mikroskopische Analysen folgendes Ergebnis hatten:

- Eine Byctiscus betulae-Blattrolle auf Linden kann aus 1-7 Blättern bestehen.

- Die Technik des Einrollens der Blattrollen ist die gleiche wie bei Weinreben.

- Die Zahl der normalen Eier beträgt 20,51 \%, die der parasitierten Eier 31,29 \%, die Zahl des frischgeschlüpften Larven $\left(\mathrm{L}_{\mathrm{n}}\right)$ ergab 20,52 \%, die der Larven $\mathrm{L}_{1} 20,14 \%$, während $\mathrm{L}_{2}$ bei $7,55 \%$ lag (Fig. 3);

- Eine Blattrolle enthält im Durchschnitt 6,31 Eier.

- Das Verhältnis zwischen normalen Eiern einerseits und parasitierten Eiern andererseits liegt bei 39,58 \% zu 60,42\% (1: 1,5).

Anhand des vorliegenden Schrifttums ließ sich feststellen, dass das Puppenstadium des Rebenstechers selten untersucht wurde; deshalb haben wir uns in den Jahren 2000, 2001 und 2002 besonders diesem Aspekt zugewandt.

Tab. 2: Analyse der Blattrollen-Bildung von Byctiscus betulae.

\begin{tabular}{|c|c|c|c|c|c|c|c|c|c|c|}
\hline & 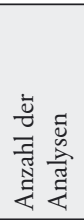 & 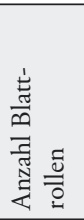 & 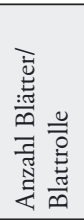 & 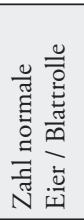 & 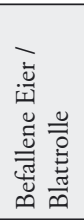 & 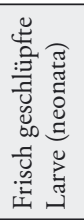 & 5 & $\Im^{2}$ & $n^{\infty}$ & 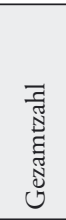 \\
\hline Anzahl & 30 & 766 & $1-5$ & 534 & 392 & 179 & 1274 & 898 & 6 & 3283 \\
\hline$\%$ & & & & 16,26 & 11,98 & 5,45 & 38,82 & 27,35 & 0,18 & 100 \\
\hline
\end{tabular}




\section{Quantitative Darstellung des Befalls bei Rebsorten in Form von "Zigarren" und Erwachsenen Byctiscus betulae L.}

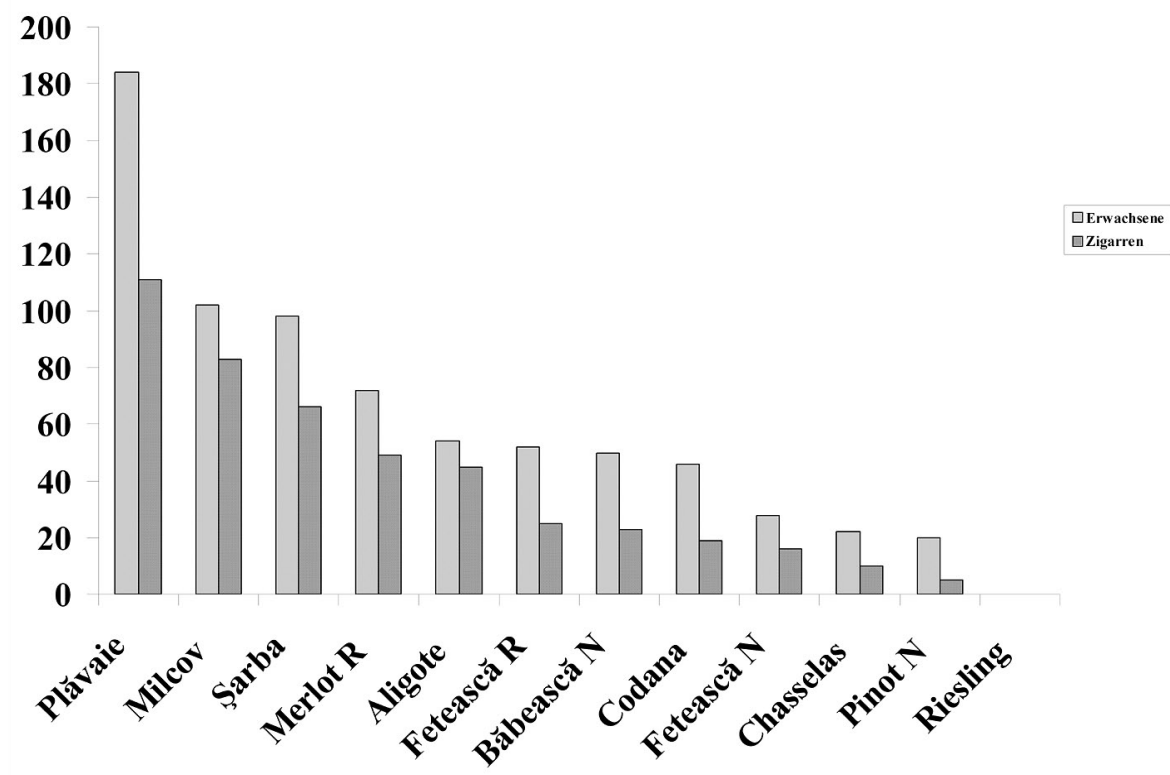

Fig. 1: Zur Untersuchung des Ei-Larven-Stadiums auf dem Weingut Odobeşti (Weinberg Şarba) im Jahr 2000 wurde am 05. und 25.05.2000 biologisches Belegmaterial zu unserem Untersuchungsobjekt gesammelt.

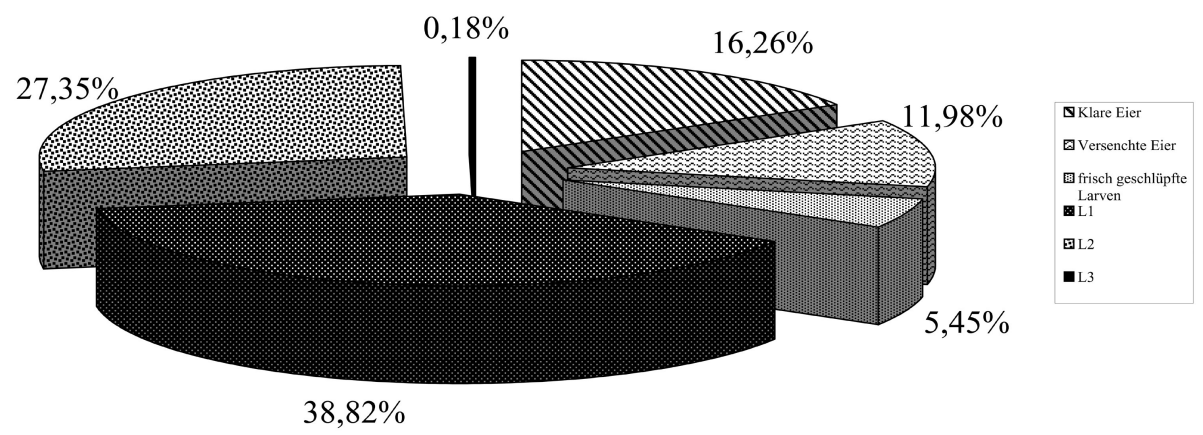

Fig. 2: Zahlenverhältnis der Entwicklungsstadien von Byctiscus betulae an Weinstöcken (Jahr 2000).

Aus der Fachliteratur geht hervor, dass die Larven $\mathrm{L}_{2}$ und $\mathrm{L}_{3}$ beim Abfallen der Blattrollen auf die Erde in den Boden eindringen und sich zwischen Juni und Oktober desselben Jahres verpuppen. 

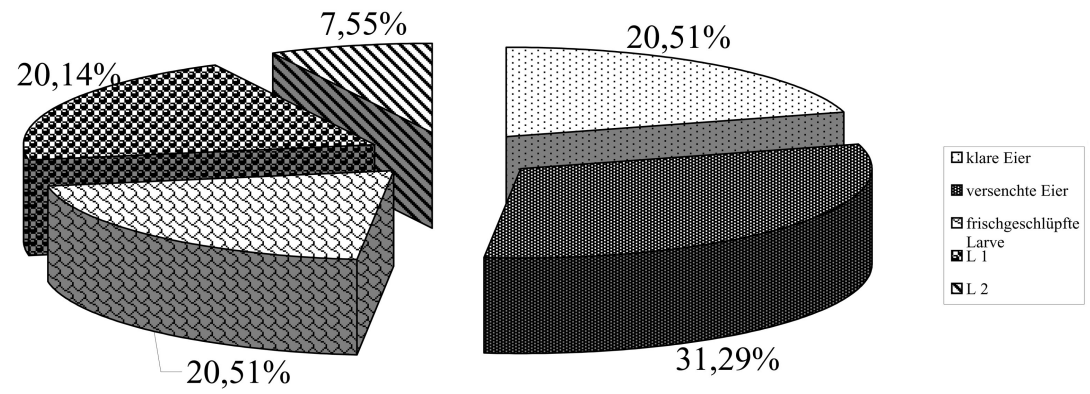

Fig. 3: Zahlenverhältnis der Entwicklungsstadien Ei-Larve von Byctiscus betulae an Linden (Jahr 2000).

Tab. 3: Analyse der Byctiscus betulae-Blattrollen: Ei, frischgeschlüpfte Larve $\left(\mathrm{L}_{\mathrm{n}}\right), \mathrm{L}_{1}, \mathrm{~L}_{2}, \mathrm{~L}_{3}$, gesammelt am 05.05.2000 an Linden.

\begin{tabular}{|c|c|c|c|c|c|c|c|c|c|c|}
\hline & 䓂 & 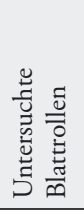 & 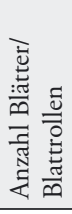 & 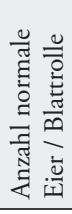 & 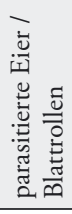 & 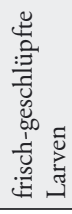 & -5 & $n^{2}$ & $\underbrace{m}$ & 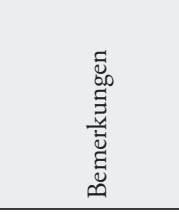 \\
\hline 1. & $11 \mathrm{Mai}$ & 5 & $2-3$ & 8 & - & 11 & - & - & - & - \\
\hline 2. & 10 Juli & 19 & $1-5$ & 6 & 23 & - & 48 & 21 & - & - \\
\hline 3. & 10 Juli & 20 & $1-7$ & 43 & 64 & 46 & 8 & - & - & $\begin{array}{l}\text { im Kühlschrank } \\
\text { aufbewahrt }\end{array}$ \\
\hline 4. & Total & 44 & $1-7$ & 57 & 87 & 57 & 56 & 21 & - & \\
\hline
\end{tabular}

Tab. 4: Übersicht über die Byctiscus betulae-Blattrollen: Ei, frischgeschlüpfte Larve $\left(\mathrm{L}_{\mathrm{n}}\right), \mathrm{L}_{1}, \mathrm{~L}_{2}, \mathrm{~L}_{3}$ an Linden (2000).

\begin{tabular}{|c|c|c|c|c|c|c|c|c|c|}
\hline & 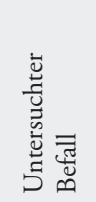 & 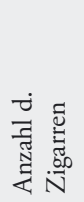 & 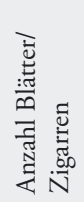 & 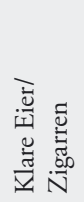 & 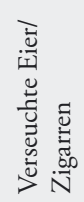 & 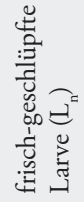 & 5 & $\Theta^{2}$ & r \\
\hline 1. & Anzahl & 44 & $1-7$ & 57 & 87 & 57 & 56 & 21 & - \\
\hline 2. & $\%$ & & & 20,51 & 31,29 & 20,51 & 20,14 & 7,55 & \\
\hline
\end{tabular}

Im Jahr 2000 haben wir 65 Erdproben an der Rebensorte Aligoté in Odobeşti (auf dem Şarba-Berg) entnommen, ohne eine einzige Byctiscus betulae-Puppe zu finden. Neben zahlreichen für die Weinkultur typischen Wirbellosen wurden lediglich drei erwachsene Rebenstecher gefunden.

Die gleiche Probe ergab in den ersten 6 Monaten des Jahres 2002 dasselbe negative Resultat. 
Am 3. Juli 2001 wurden vier Stellen zur Vorpuppen-Entwicklung eingerichtet, und zwar: Bett I mit 300 Blattrollen; Bett II: 276 Blattrollen; Bett III: 106 Blattrollen; Bett IV: 106 Blattrollen. Die Funde vom 07.06.2001ergaben in der Summe 788 Blattrollen.

Die Versuchsstellen im Freien wurden zwischen dem 12. und 13. September überprüft, ohne jedoch die in Gang befindlichen Entwicklungen zu stören.

Am 30. und 31. Oktober 2001 wurden 2 der 4 Anlagen (I, III) geöffnet, doch auch diesmal fielen die Laboranalysen negativ aus.

Die beiden restlichen Nester, die Proben II und IV, wurden am 19.03.2002 ausgehoben; sie enthielten nur 14 Larven $\mathrm{L}_{2}$ und 4 Imagines.

Tab. 5: Schlußfolgerungen der Untersuchungen Byctiscus betulae aus dem Jahr 2002.

\begin{tabular}{|c|c|c|}
\hline & Datum & Anmerkungen \\
\hline 1. & 25.06.2002 & Einführung der Blattrollen in einen mittleren Feuchtigkeitsbereich von 53 - 55 \%. \\
\hline 2. & 05.07.2002 & Die notwendige Bodenfeuchtigkeit wird gesichert. \\
\hline 3. & 11.07.2002 & Die notwendige Bodenfeuchtigkeit weiterhin gesichert. \\
\hline 4. & 17.07.2002 & Es werden die ersten vier Larven $\left(\mathrm{L}_{3}\right)$ gesichtet. \\
\hline 5. & 22.07.2002 & $\begin{array}{l}\text { Es können } 8 \text { große Larven } \mathrm{L}_{3} \text { verzeichnet werden, von denen zwei sich bereits Puppen- } \\
\text { höhlungen gebaut hatten. }\end{array}$ \\
\hline 6. & 24.07.2000 & Sicherung des Bodenfeuchtigkeit. \\
\hline 7. & 30.07.2002 & $\begin{array}{l}\text { Sicherung der erforderlichen Bodenfeuchtigkeit. Die Entwicklung der Insekten erreicht } \\
\text { das letzte Stadium. }\end{array}$ \\
\hline 8. & 07.08.-13.08.2000 & 12 große Larven $\mathrm{L}_{3}$ haben sich im Feuchtebereich von $55 \%$ angesiedelt. \\
\hline 9. & 19.08.2002 & Unveränderte Sachlage. \\
\hline 10. & 20.08.2002 & In der Puppen-Höhlung wird die 1. Puppe festgestellt, dazu zahlreiche Larven $\mathrm{L}_{3}$. \\
\hline 11. & 21.08.2002 & Es werden weitere zwei Puppen entnommen. \\
\hline 12. & 22.08.2002 & $\begin{array}{l}\text { Vier lebendige Puppen und zahlreiche Larven } \mathrm{L}_{3} \text { werden für fotografische Aufnahmen ent- } \\
\text { nommen. }\end{array}$ \\
\hline 13. & 23.08.2002 & Es sind abermals zahlreiche Puppen und Larven $\mathrm{L}_{3}$ zu vermerken. \\
\hline 14. & 26.08.2002 & In der Puppen-Höhlung tritt die erste Imago auf. \\
\hline 15. & 24.09.2002 & Neben Erwachsenen befinden sich lebende Puppen. \\
\hline 16. & 05.11 .2002 & Im Untersuchungsfeld finden sich lebende Larven und Puppen. \\
\hline 17. & 14.01 .2003 & Zwei Puppen-Höhlungen mit Puppen werden separiert sowie 5 Larven $\mathrm{L}_{3}$. \\
\hline
\end{tabular}

Im Jahr 2002 entschieden wir uns für Laboruntersuchungen, um zu Puppen von Byctiscus betulae zu gelangen. Auf Grund des Blattrollen-Ertrages, dessen Stückzahl am 23.05.2002 564 Exemplare ergeben hatte, richteten wir am 12.06.2002 einen FeuchtigkeitsGradienten ein, in den 100 Blattrollen mit Larven $\mathrm{L}_{2}$ und $\mathrm{L}_{3}$ eingeführt wurden. Die Beobachtungen im folgenden Zeitabschnitt enthält die nachfolgende Tabelle 5.

Fazit: Die erste Puppe von Byctiscus betulae wurde erst als Folge unseres Laborversuchs über die Einrichtung eines Feuchtigkeitsgradienten greifbar; die erste Imago wurde innerhalb einer Woche nach der ersten Puppe erzielt. Die Byctiscus betulae-Puppe ist stenohyger (feuchtigkeitsabhängig), da sie nur bei einem Feuchtigkeitswert von 50-55 \% aufgetreten ist. 


\section{Schlußfolgerungen}

1. Das biologische Belegmaterial der Jahre 2000-2002 erlaubt uns, nun das Schema des biologischen Zyklus von Byctiscus betulae für jedes Jahr zu erarbeiten (Tabelle 6).

- Der Rebstecher ist in den Wintermonaten Januar bis März im Imaginalstadium anzutreffen, das in der Puppenhülle oder einfach im Boden überwintert.

- In der ersten April-Dekade treten auf Rebenblättern die ersten Imagines auf, die sich bis zur Geschlechtsreife ernähren und dann kopulieren.

- Im Mai beginnt die Eiablage und die erste Blattrollen-Bildung; Wir nehmen mit Sicherheit an, dass die früheste Eiablage in der ersten Mai-Dekade stattfindet;

- Aus den herangereiften Eiern schlüpfen nach 8-10 Tagen die ersten Larven. Das Larven-Stadium dauert von Mai bis August (d. i.: $\left.\mathrm{L}_{1}, \mathrm{~L}_{2}, \mathrm{~L}_{3}\right)$.

- Das Puppenstadium erstreckt sich von Mitte August bis Ende September, dem Zeitpunkt, an dem die überwinternde Puppe in ihrer Höhlung erwachsen wird. Sie überlebt längere oder kürzere Zeit, je nach physikalischer Gegebenheit, und setzt im April des folgenden Jahres ihren biologischen Zyklus fort.

2. Aufgrund unserer Daten liess sich die Dauer der Entwicklungsstadien von Byctiscus betulae für das Jahr 2002 festlegen.

3. Die stufenweise Staffelung des biologischen Zyklus von Byctiscus betulae für das Jahr 2002 war folgende:

- Am 03.05. des Jahres trat das erste Ei in der grünen Blattrolle auf.

- 23.05. wird die 1. Blattrolle mit normalen Eiern eingesammelt.

- Am 25.05. werden daraus die 1. frischgeschlüpfen Larven entnommen.

- Die Dauer der Embryogenese kann mit 3-4 Tagen angenommen werden.

- Dasselbe Studienmaterial wird bis zum 17.07. im Auge behalten; dann treten die ersten Larven $\mathrm{L}_{3}$ auf. Für das Larvenstadium wird eine Dauer von 52 Tagen angesetzt (inklusive einer Abweichung von plus/minus 2 Tagen).

- Die Beobachtungen gehen bis zum 19.08. weiter, an dem die erste Puppe erscheint, nämlich 32 Tage nach dem Auftreten der der $\mathrm{L}_{3}$.

- Das Erscheinen der Erwachsenen wurde am 02.09. verzeichnet, nach einem Intervall von 14 Tagen.

- Die Erwachsenen starben am 12.09.2002 nach 10 Tagen Haltung (ohne Nahrung und Wasser).

Tab. 6: Stufenweise Staffelung des biologischen Zyklus von Byctiscus betulae für das Jahr 2002.

\begin{tabular}{|llll|}
\hline & Datum & Feststellungen & Dauer des Stadiums \\
1. & 03.05 .2002 & erstes Ei in der grünen Blattrolle & \\
2. & 23.05 .2002 & Blattrollen mit frischen normalen Eiern & 3-4 Tage \\
3. & 26.05 .2002 & Larva neonata & 52 Tage \\
4. & 17.07 .2002 & Larve $\mathrm{L}_{3}$ & 32 Tage \\
\hline 5. & 19.08 .2002 & Puppe & 14 Tage \\
6. & 02.09 .2002 & lebende Imago & 10 Tage \\
\hline 7. & 12.09 .2002 & tote Imago & \\
\hline
\end{tabular}




\section{Literatur}

Antonie, I. 2002: Das numerisch relative Vorkommen des Zigarrendrehers bei Rebstöcken in den Böden der Weinpflanzungen. - Lucrările Conferinței „Ştiințe, Procese şi Tehnologii Agro-Alimentare”, Ed. Univ. „Lucian Blaga” Sibiu, 180-184, Sibiu.

Balachowsky, A. S. \& Hoffmann, A. 1963: Famille des Attelabidae. - Entomologie Appliquée a l'Agriculture 1: 1202-1237.

Becker, H. 1954: Beiträge zur Kenntnis des Rebstichlers (Byctiscus betulae L.). - Beiträge zur Entomologie, Berlin 4 (2): 158-172.

Bielz, E. A. 1851: Systematisches Verzeichniss der Käfer Siebenbürgens. - Verhandlungen und Mitteilungen des Siebenbürgischen Vereins für Naturwissenschaften, Hermannstadt 2 (2):18-43.

DaAnje, A. 1964: Über die Ethologie und Blattrolltechnik von Deporaus betulae L. und ein Vergleich mit den anderen blattrollenden Rhynchitinen und Attelabinen (Coleoptera, Attelabinae). - Verhandelingen der Koninklyke Nederlandse Akademie van Wetenschappen, Afd. Natuurkunde, Tweede Reeks 56: 1-215.

Dieckmann, L. 1974: Beiträge zur Insektenfauna der DDR: Coleoptera, Curculionidae (Rhinomacerinae, Rhynchitinae, Attelabinae, Apoderinae). - Beiträge zur Entomologie, Berlin 24 (1/4): 5-54.

Fuss, C. 1869: Verzeichnis der Käfer Siebenbürgens nebst Angabe ihrer Fundorte. - Archiv des Vereins für Siebenbürgische Landeskunde, Kronstadt 7 (3): 335-490.

Косн, K. 1992: Die Käfer Mitteleuropas. Ökologie. - Goecke \& Evers Verlag, Krefeld.

Petri, K. 1912: Siebenbürgens Käferfauna auf Grund ihrer Erforschung bis zum Jahr 1911. - Jos. Drotleff, Hermannstadt.

Prell, H. 1925: Der Trichterwickel des Birkenblattrollers. - Die Naturwissenschaften 13 (30): 1-15.

PreLl, H. 1924: Die biologischen Gruppen der deutschen Rhynchitiden. - Zoologischer Anzeiger, Jena 61 (7/8): 153-170.

Prell, H. 1925: Die Trichterrolle des Ahornblattrollers. Biologisches und Taxonomisches über einen sehr bemerkenswerten Rüsselkäfer. - Zeitschrift für Morphologie und Ökologie der Tiere, Abt. A 3 (5): 685-703.

Scherf, H. 1964: Die Entwicklungsstadien der mitteleuropäischen Curculioniden (Morphologie, Bionomie, Ökologie). - Abhandlungen der Senckenbergischen naturforschenden Gesellschaft, Frankfurt 506: $1-335$.

Zuppa, A.; Ossela, G. \& Biondi, S. 1994: Parental care in Attelabidae (Coleoptera, Curculionoidea). - Ethology Ecology \& Evolution, Special Issue 3: 113-118.

ZwöLFeR, H. 1969: Rüsselkäfer mit ungewöhnlicher Lebensweise. - Mitteilungen der Schweizerischen Entomologischen Gesellschaft 42 (3): 185-196, Koleopterologische Rundschau.

Subject editor: H. H. DATHE

\section{Anschrift der Verfasserinnen:}

Dr. Iuliana Antonie

Fakultät für Agrarwissenschaften

Lebensmittelindustrie und

Umweltschutz in Sibiu/Hermannstadt

Str. Dr. Ion Rațiu, nr. 7-9

RO-550012 Sibiu

iuliana_antonie@yahoo.com
Prof. Dr. Irina Teodorescu

Fakultät für Biologie

Universität Bukarest

Spl. Independenței 91-95

sector 5

RO-050095 Bukarest

iteodorescu@bio.unibuc.ro
Prof. Dr. Letiția Oprean

Fakultät für Agrarwissenschaften

Lebensmittelindustrie und Umweltschutz in Sibiu / Hermannstadt

Str. Dr. Ion Rațiu, nr. 7-9

RO-550012 Sibiu

saiapm@ulbsibiu.ro 